\title{
Effets des facteurs hydriques et osmotiques sur la croissance des talles de blé
}

\author{
JP Gaudillère *, MO Barcelo \\ INRA INA PG, laboratoire de chimie biologique et de photophysiologie, 78850 Thiverval-Grignon, France
}

(Reçu le 13 mars 1989; accepté le 18 mars 1990)

\begin{abstract}
Résumé - Les paramètres principaux régissant la croissance en longueur des talles de blé : potentiel hydrique, osmotique et de turgescence, ainsi que certains constituants de l'osmolarité ont été étudiés en conditions contrôlées et au champ. L'influence de ces paramètres sur la régression des talles observée généralement au cours de la montaison a été recherchée. On observe une compartimentation stricte de l'eau entre les zones adultes et en croissance d'un limbe. Le potentiel hydrique des zones en croissance est peu dépendant des fluctuations des conditions agroclimatiques. Le maître brin (MB) et la talle $3\left(T_{3}\right)$ présentent des stratégies différentes de maintien de leur croissance en cas de déficit hydrique : le maître brin peut garder plus longtemps une turgescence positive avec un potentiel osmotique systématiquement plus bas que celui de la $T_{3}$. Par contre, celle-ci peut croître à des pressions de turgescence plus faibles, car le potentiel seuil de croissance est plus faible que celui du maître brin. Ces caractéristiques peuvent contribuer à l'homogénéisation de la taille des talles dont la date de début d'allongement varie avec le rang. La population de $\mathrm{T}_{3}$ qui régresse au cours de la montaison n'a pas pu être caractérisée précocement par les paramètres hydriques impliqués dans les modèles décrivant l'allongement. On note cependant que les talles en régression sont plus pauvres en sucres et plus riches en nitrates.
\end{abstract}

Triticum aestivum / potentiel hydrique / potentiel osmotique / turgescence

Summary - The effects of water potential and osmotic potential on wheat tiller growth. The main growth parameters, water, osmotic and turgor potential of wheat tillers are studied in controlled and field-grown plants. The objective of the study was to analyse the decline of the tiller during shoot elongation.

Between the growing and the adult part of blades, there is a compartmentation of water. Water potential of the growing parts is not so dependent on variations in the environment.

There are different biomechanical properties between the main and the 3rd tiller. The osmotic potential of the main tiller is lower than that of the 3rd tiller. Accordingly, the main tiller can maintain a positive turgor for longer periods. The turgor threshold of the 3rd tiller is lower and requires a lower turgor to maintain the same relative growth rate as the main tiller. These properties could lead to growth compensation between tillers.

The 3rd tiller population which declines during the fast growing phase cannot be sorted at an early stage with the growth parameters we studied, as these parameters change when the decline is obvious. At that time, a decrease in sugar content and a relative increase in nitrate content are measured in declining tillers.

Triticum aestivum / water potential / osmotic potential / turgor / stem elongation

\section{INTRODUCTION}

L'occupation de l'espace par une culture de blé est déterminée par le nombre de plantes installées et par le nombre de talles que ces plantes sont capables de développer. La densité de peuplement des talles varie donc pendant le tallage et dépend du rythme de leur apparition. Lorsque l'occupation est réalisée, la taille individuelle des plantes, exprimée par exemple en poids de matière sèche, est négativement corrélée à la densité du peuplement (nombre de plantes par unité de surface). Cette corrélation permet une certaine optimisation de l'utilisation de l'espace et des ressources nutritionnelles (Silverstown, 1982).

\footnotetext{
* Correspondance et tirés à part. Adresse actuelle : INRA, centre de Bordeaux, station de physiologie végétale, BP 131, 33140 Pontde-la-Maye, France
} 
Cependant, à forte densité de peuplement, on observe des arrêts de croissance et des régressions de talles pendant la montaison. Westoby (1984) a observé que les compétitions entre individus au sein d'un peuplement en croissance conduisent à la disparition de certains d'entre eux. L'évolution de la densité du peuplement (auto-éclaircissage) suit une loi générale, valable pour un grand nombre d'espèces et pour différentes conditions d'environnement où s'exerce cette compétition. Chez le blé, ainsi que chez la plupart des graminées fourragères, l'autoéclaircissage entraîne rarement la disparition entière de plantes. La régulation de la densité se fait au niveau du nombre de talles.

La régression des talles, telle qu'elle a été caractérisée par Masle-Meynard (1981 a et b), résulte principalement d'une compétition entre les différentes zones en allongement de la plante vis-à-vis des principaux facteurs trophiques : eau, carbone ou azote. Toutefois ces 2 derniers facteurs ne semblent pas limitants dans les conditions culturales habituelles (Kemp et Blacklow, 1980, Kemp, 1981 a et b) et ne sont probablement pas responsables de la différence de vitesse de croissance entre talles (Kemp et Whingwiri, 1980). Les paramètres hydriques pourraient donc avoir un rôle déterminant dans l'autoéclaircissage en cours de montaison.

Le rôle de l'alimentation hydrique et des propriétés mécaniques des parois peut être étudié à l'aide du modèle de Lockhart (1965), décrivant l'allongement des cellules ou des tissus (Taiz, 1984, Tomos, 1985).

L'allongement des cellules sous l'action de la pression de turgescence est déterminé par leurs propriétés mécaniques viscoélastiques. La première équation de Lockhart (1965) traduit la relation entre la vitesse d'augmentation relative du volume cellulaire $(V)$ et la différence de pression $(T)$ entre la paroi et les cellules :

$$
(1 / V) \cdot d V / d t=m \cdot(T-Y)
$$

$Y$ est le seuil de pression de turgescence audelà duquel se manifeste un allongement.

$m$ est un coefficient de proportionnalité qui exprime l'extensibilité de la paroi.

Ces 2 paramètres évoluent avec les changements de structure de la paroi au cours de la maturation des tissus. Ils peuvent également varier à court terme, en particulier, en réponse à des modifications du $\mathrm{pH}$ apoplasmique (Matthews et al, 1984; Fry, 1986; Barlow, 1986).

La relaxation de la paroi sous l'effet de la pression de turgescence s'accompagne d'une diminution du potentiel hydrique, ce qui crée le gradient nécessaire à l'entrée d'eau dans les cellules et permet leur changement de volume. La seconde équation de Lockhart (1965), reformulée par Cosgrove (1986) décrit le mécanisme d'entrée de l'eau :

$$
(1 / V) \cdot \mathrm{d} V / \mathrm{d} t=L \cdot\left(s \cdot P_{i}-T\right)
$$

L est la conductance hydraulique volumétrique $\left(\mathrm{s}^{-1} \mathrm{MPa}^{-1}\right)$.

$s$ est le coefficient de réflection membranaire pour les solutés.

$P_{i}$ est la différence de pression osmotique entre l'intérieur des cellules et l'apoplasme.

L'entrée d'eau tend à diminuer $P_{i}$, une accumulation active de molécules susceptibles d'augmenter le potentiel osmotique est donc nécessaire pour entretenir le processus d'allongement en maintenant la turgescence. La nature des molécules reflète des caractéristiques métaboliques du processus d'allongement (Handa et al, 1983). Ce modèle primitivement développé pour décrire la croissance de cellules isolées a été appliqué à des tissus végétaux plus complexes. Ces équations sont donc empiriques mais elles permettent cependant une analyse des paramètres physiques qui gouvernent la croissance (Tomos, 1985).

Ce travail a été entrepris pour étudier les relations entre quelques facteurs biophysiques et biochimiques et la croissance des talles de blé au cours du tallage et de la montaison. Nous avons déterminé les potentiels hydrique et osmotique, ainsi que la nature des principales molécules responsables de ce potentiel, sur des plantes cultivées au champ ou en conditions contrôlées. Les caractéristiques des maîtres brins et des $T_{3}$ ont été comparées pour vérifier si elles peuvent contribuer à expliquer leur comportement au champ.

\section{MATÉRIEL ET MÉTHODES}

Les observations ont été effectuées sur blé tendre, Triticum aestivum, variété Fidel, dont les potentialités de tallage sont importantes. 


\section{Expériences en conditions de culture contrôlées}

\section{Conditions de culture}

Les grains sont mis à germer sur vermiculite à l'obscurité, à $20^{\circ} \mathrm{C}$ et à saturation d'humidité. Lorsque les plantules atteignent le stade foliaire 1,5 , la vernalisation est obtenue par un traitement de $12 \mathrm{j}$ à $4{ }^{\circ} \mathrm{C}$ sous une photopériode de $15 \mathrm{~h}$. Puis les plantes sont cultivées sur une solution nutritive complète, aérée, contenant $12 \mathrm{mmol}$ d'azote (Coïc et Lesaint, 1973). La température est de $20{ }^{\circ} \mathrm{C}$, l'humidité relative de $70 \%$, la photopériode est de $15 \mathrm{~h}$, la densité de flux de photons est de $180 \mu \mathrm{mol} \cdot \mathrm{m}^{-2} \cdot \mathrm{s}^{-1}$. Les mesures en conditions contrôlées ont porté sur des plantes âgées d'environ $30 \mathrm{j}$ et dont le stade foliaire varie entre 7 et 9 pour $\mathrm{MB}$ et 3 à 5 pour $\mathrm{T}_{3}$.

\section{Traitements expérimentaux}

Deux types de traitements induisant une contrainte hydrique au laboratoire ont été appliqués :

\section{Traitement 1}

Un assèchement progressif des racines est réalisé par une diminution continue du volume de la solution nutritive à une vitesse moyenne de $25 \%$ par $h$. Le traitement est réalisé en milieu de photopériode, sur des plantes transpirant activement. Une heure après l'arrêt complet de l'allongement, la dernière feuille de MB et celle de $\mathrm{T}_{3}$ sont prélevées et les tissus engainés (limbe et gaine en croissance) et dégainés (partie adulte du limbe) sont séparés. Ces échantillons sont soumis aux analyses faisant l'objet d'une description ultérieure.

\section{Traitement 2}

Ces segments de limbes en croissance sont excisés et placés à pH 5,6, dans une solution tamponnée (tampon acétate $10 \mathrm{mmol} \cdot \mathrm{I}^{-1}$ ) contenant $10 \mathrm{mmol}$ de $\mathrm{KCl}$ (Vesper, 1985). La pression osmotique du milieu est augmentée par paliers de $100 \mathrm{m0s} \mathrm{kg}^{-1}$. L'osmoticum utilisé est le mannitol. À l'arrêt de l'allongement, (on décide que cet arrêt a eu lieu après une $h$ de croissance nulle), la différence de potentiel osmotique entre la solution et le contenu cellulaire représente alors le potentiel seuil $Y$ (Cleland, 1971).

\section{Mesure de l'allongement}

Pendant ces traitements, l'allongement est mesuré en permanence avec une résolution de $45 \mu \mathrm{m}$ par un cap- teur de déplacement linéaire (Iffelec, Paris) attaché à la feuille et exerçant sur celle-ci une force de $0,1 \mathrm{~N}$.

\section{Conditions de culture au champ}

Le blé cultivé au champ est semé à Grignon en novembre 1984 à 2 densités, 150 et 300 plantes par $\mathrm{m}^{2}$. À la densité la plus forte on constate la régression des dernières talles apparues. Les plantes ont bénéficié de 3 apports d'azote $(240 \mathrm{~kg} / \mathrm{ha})$ entre le début du tallage et la fin de la montaison, de façon à assurer une alimentation azotée non limitante. Des prélèvements de $M B$ et de $T_{3}$ ont été effectués pendant le tallage et la montaison à 7 dates correspondant aux sommes de température suivantes : $611,695,755$, 833,900, 971 et 1112 degrés-jours exprimés en base $0^{\circ} \mathrm{C}$ à partir de la levée. Le stade foliaire du MB est passé de 5 à 11 pendant la durée des observations.

\section{Méthodes d'analyse}

Le potentiel hydrique des tissus prélevés est mesuré à l'aide d'une bombe à pression, selon la méthode de Scholander et al (1965). Les échantillons sont ensuite congelés à $-15^{\circ} \mathrm{C}$ puis décongelés à $5{ }^{\circ} \mathrm{C}$ : leur pressage dans une seringue de $1 \mathrm{ml}$ permet alors d'extraire un jus ( $10 \mu$ l environ). Ce jus est séparé après dilution en plusieurs fractions aliquotes pour les analyses ultérieures.

La concentration osmotique est obtenue par mesure de l'abaissement de la température de congélation, à l'aide d'un microosmomètre (Roebling). Nous avons déterminé, par la méthode de Katz et Avron (1985), que le suc apoplasmique représente environ $15 \%$ du volume du jus foliaire. Comme nous n'avons pas pu collecter de l'eau apoplasmique, nous avons admis que sa concentration osmotique moyenne est 70 mosm (Cosgrove et Cleland, 1983). Ces valeurs permettent d'estimer, la concentration osmotique intracellulaire dans les échantillons. Le calcul de la pression osmotique correspondante est ensuite effectué selon la loi de Van't Hoff. La turgescence est enfin obtenue par soustraction des valeurs mesurées des potentiels hydriques et osmotiques.

Une partie aliquote du jus foliaire est séchée sous vide, puis reprise dans la pyridine. Des dérivés silylés des sucres solubles et du malate sont ensuite réalisés et dosés par chromatographie en phase gazeuse (Sweeley, 1965).

Les nitrates sont dosés par la méthode colorimètrique de Cataldo et al (1975). Le potassium est dosé par spectrophotométrie de flamme. 


\section{RÉSULTATS}

\section{Caractéristiques hydriques, le long d'une feuille en cours d'allongement, en conditions de culture contrôlées}

\section{Potentiels hydrique, osmotique et de turgescence}

Les caractéristiques hydriques de $M B$ et $T_{3}$ ne sont pas différentes (tableau I). Après $8 \mathrm{~h}$ d'éclairement, les tissus engainés en cours d'allongement ont un potentiel hydrique supérieur de 0,4 à $0,5 \mathrm{MPa}$ à celui des tissus dégainés. Cet écart se maintient en fin de nuit. Le potentiel osmotique ne présente pas de gradient le long de la feuille et est de 1,5 $\pm 0,1 \mathrm{MPa}$ pour l'ensemble des mesures. Par conséquent, il existe au sein de la feuille un gradient permanent de

Tableau I. Potentiels hydrique et osmotique et pression de la turgescence dans la dernière feuille du maître brin (MB) et de talle $3\left(T_{3}\right)$ de plantes de blé en fin de nuit et après $8 \mathrm{~h}$ de lumière. MB $\mathrm{E}$ : maître brin partie du limbe engainée; MB D : maître brin partie du limbe dégainée; $T_{3} E$ : talle 3 partie du limbe engainée; $T_{3} D$ : talle 3 partie du limbe dégainée. \pm intervalle de confiance à $5 \%, 4$ répétitions en fin de nuit (a. $b$ test de Student apparié significativement différent) et 14 répétitions après $8 \mathrm{~h}$ de jour ( $c, d$ test de Student apparié significativement différent).

\begin{tabular}{|c|c|c|}
\hline Talle & Fin de nuit & $8 \mathrm{~h}$ de lumière \\
\hline & \multicolumn{2}{|c|}{ Potentiel hydrique (MPa) } \\
\hline \multirow[t]{2}{*}{$\begin{array}{l}\text { MB E } \\
\text { MB D } \\
T_{3} E \\
T_{3} \quad D\end{array}$} & $\begin{array}{l}-0,12 \pm 0,05^{a} \\
-0,46 \pm 0,17^{b} \\
-0,12 \pm 0,08^{a} \\
-0,49 \pm 0,08^{b}\end{array}$ & $\begin{array}{l}-0,27 \pm 0,12^{c} \\
-0,94 \pm 0,18^{d} \\
-0,48 \pm 0,24^{c} \\
-0,87 \pm 0,18^{d}\end{array}$ \\
\hline & \multicolumn{2}{|c|}{ Potentiel osmotique (MPa) } \\
\hline $\begin{array}{l}\text { MB E } \\
\text { MB D } \\
T_{3} E \\
T_{3} \quad D\end{array}$ & $\begin{array}{l}-1,41 \pm 0,19^{a} \\
-1,34 \pm 0,09 a \\
-1,36 \pm 0,16^{a} \\
-1,33 \pm 0,13^{a}\end{array}$ & $\begin{array}{l}-1,81 \pm 0,29^{c} \\
-1,56 \pm 0,29^{c} \\
-1,50 \pm 0,13^{c} \\
-1,54 \pm 0,08^{c}\end{array}$ \\
\hline $\mathrm{T}_{3} \mathrm{D}$ & \multicolumn{2}{|c|}{ Pression de turgescence ( $\mathrm{MPa}$ ) } \\
\hline $\begin{array}{l}M B E \\
M B D \\
T_{3} E \\
T_{3} D\end{array}$ & $\begin{array}{l}1,29 \pm 0,21^{a} \\
0,93 \pm 0,22^{b} \\
1,23 \pm 0,21^{a} \\
0,85 \pm 0,13^{a}\end{array}$ & $\begin{array}{l}1,64 \pm 0,36^{c} \\
0,62 \pm 0,20^{d} \\
1,03 \pm 0,27^{c} \\
0,67 \pm 0,17^{d}\end{array}$ \\
\hline
\end{tabular}

pression de turgescence. Au cours de la journée, la pression de turgescence varie avec le potentiel hydrique. Elle reste élevée dans nos conditions, en particulier dans les tissus engainés.

\section{Composition osmotique du suc cellulaire foliaire}

L'analyse de la composition osmotique ne montre pas d'effet du rang des talles et la comparaison porte sur les zones des $\mathrm{MB}$ et $\mathrm{T}_{3}$ en allongement et celles ayant terminé leur croissance (tableau II). Les composés dosés sont responsables de $77 \%$ de l'osmolarité totale. Les ions $\mathrm{K}^{+}$et $\mathrm{No}_{3}{ }^{-}$sont les responsables majeurs de cette osmolarité. Ils sont en concentration équivalente dans les tissus engainés et dégainés. Même après $8 \mathrm{~h}$ d'éclairement, les quantités de sucres solubles restent faibles. II s'agit essentiellement de glucose et de fructose, répartis en concentrations équivalentes au sein des feuilles observées. Par contre, les zones en croissance sont plus riches en malate, seul anion organique dosé, mais plus paurres en saccharose, que tissus adultes.

\section{Caractéristiques hydriques des feuilles à l'arrêt de croissance provoqué par une contrainte hydrique}

Les plantes âgées de $30 \mathrm{j}$ sont soumises aux différents traitements décrits dans la section Matériel et Méthodes. Les vitesses d'élongation des plantes avant le traitement étaient toujours supérieures à $1,5 \mathrm{~mm} \cdot \mathrm{h}^{-1}$.

Tableau II. Principaux constituants osmotiques $\left(\mathrm{mmol} \cdot \mathrm{kg}^{-1}\right) 8 \mathrm{~h}$ après le début de l'éclairement dans les feuilles en croissance de plantes de blé cultivées en conditions contrôlées. Les lettres différentes (a.b.c) indiquent des différences significatives à $5 \%$ par le test de Student apparié. Moyenne \pm l'intervalle de confiance (10 répétitions).

\begin{tabular}{|c|c|c|}
\hline Constituants & Tissu engainé & Tissu dégainé \\
\hline$K^{+}$ & $302 \pm 28$ & $334 \pm 20$ \\
\hline $\mathrm{NO}_{3}^{-}$ & $94 \pm 29$ & $68 \pm 20$ \\
\hline Fructose & $6 \pm 2$ & $7,6 \pm 4$ \\
\hline Glucose & $12,5 \pm 5$ & $12,2 \pm 6$ \\
\hline Saccharose & traces ${ }^{a}$ & $1,2 \pm 2^{c}$ \\
\hline Acide Malique & $13,5 \pm 2^{b}$ & $0,8 \pm 2^{c}$ \\
\hline
\end{tabular}


Lors du traitement 1 (tableau III), l'abaissement du niveau de la solution nutritive autour des racines provoque une diminution progressive de la vitesse d'allongement. L'arrêt complet de la croissance n'est obtenu que lorsque les racines sont totalement émergées.

On constate alors, après $1 \mathrm{~h}$ de croissance nulle, que la turgescence des parties engaînées n'est pas significativement différente de celle du témoin bien alimenté en eau; cette méthode n'est donc pas adéquate pour mesurer le potentiel seuil de turgescence $Y$. L'arrêt d'allongement est probablement dû à un autre facteur que la pression de turgescence. L'approvisionnement en eau nécessaire à l'expression de l'allongement (augmentation de volume) pourrait être, dans ces conditions, le facteur déterminant, puisque l'arrêt de croissance n'est observé qu'au moment où toutes les racines sont hors de la solution. La différence de turgescence entre parties engainées et dégainées du MB n'est pas modifiée par le traitement.

De même, les pressions osmotiques mesurées dans notre matériel (1,6 MPa en moyenne) restent constantes au cours du traitement. Enfin le $M B$ et $T_{3}$ présentent la même réponse au traitement.

Dans le traitement 2, des solutions de pression osmotique croissante sont appliquées directement sur les tissus excisés en allongement. La pression de turgescence des tissus est déterminée à l'arrêt de l'allongement. Elle est respectivement de 0,97 $\pm 0,13 \mathrm{MPa}$ pour MB et de 0,77 \pm $0,25 \mathrm{MPa}$ pour $\mathrm{T}_{3}$. La grande variabilité des pressions de turgescence à l'arrêt de croissance s'explique pour $T_{3}$ par une corrélation significative

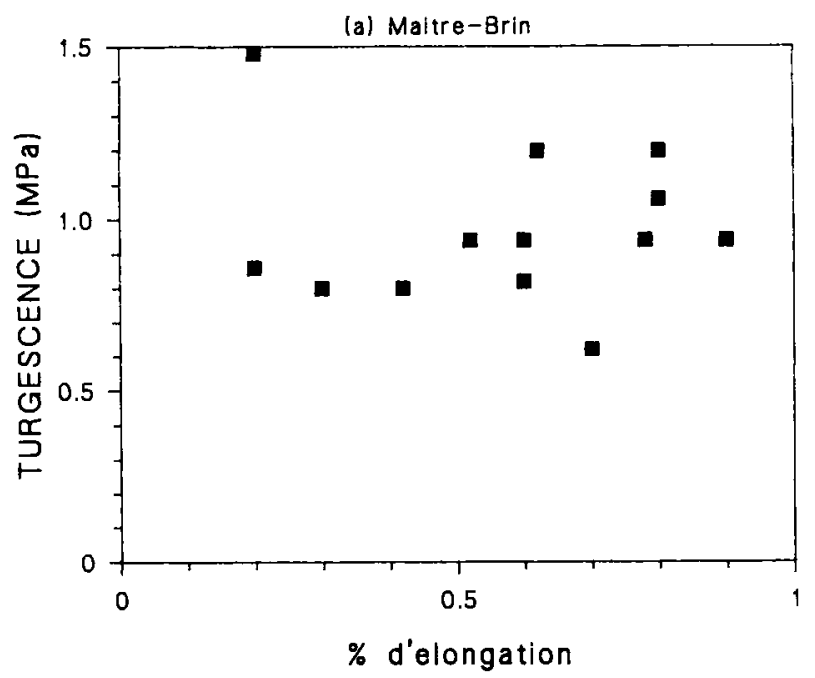

Tableau III. Effet de la réduction du niveau de la solution nutritive autour des racines sur la pression de turgescence (en $\mathrm{MPa}$ ) de la dernière feuille du maître brin (MB) et de la talle $3\left(\mathrm{~T}_{3}\right)$ ayant arrêté leur croissance (tissus engainés : $E$ et dégainés : $D$ ). Les moyennes significativement différentes ont des lettres différentes (a,b) (test de Student apparié à $5 \%, 4$ répétitions, \pm intervalle de confiance).

Pression de turgescence

Tissu prélevé Plante témoin Plante traitée à en croissance l'arrêt de croissance

$\begin{array}{lll}\text { MB E } & 1,3 \pm 0,6^{\mathrm{a}} & 1,5 \pm 0,2^{\mathrm{a}} \\ \text { MB D } & 0,5 \pm 0,5^{\mathrm{b}} & 0,4 \pm 0,2^{\mathrm{b}} \\ T_{3} E & 0,8 \pm 0,8^{\mathrm{b}} & 1,5 \pm 0,2^{\mathrm{b}} \\ T_{3} D & 0,6 \pm 0,5^{\mathrm{b}} & 0,6 \pm 0,2^{\mathrm{b}}\end{array}$

$(r=0,75 ; n=13)$ entre ce paramètre et le pourcentage de la taille finale atteinte de la feuille (fig 1b). Le potentiel seuil est d'autant plus élevé que la feuille approche sa taille finale. Par contre le MB ne présente pas cette corrélation (fig 1a); le potentiel seuil de MB ne dépend donc pas de la taille ou de l'âge de la feuille.

\section{Évolution des paramètres hydriques de $M B$ et $T_{3}$ pendant le tallage et la montaison de blés cultivés au champ}

\section{Comparaison de MB et $\mathrm{T}_{3}$}

Le tableau IV rapporte les valeurs moyennes des potentiels hydrique et osmotique mesurés

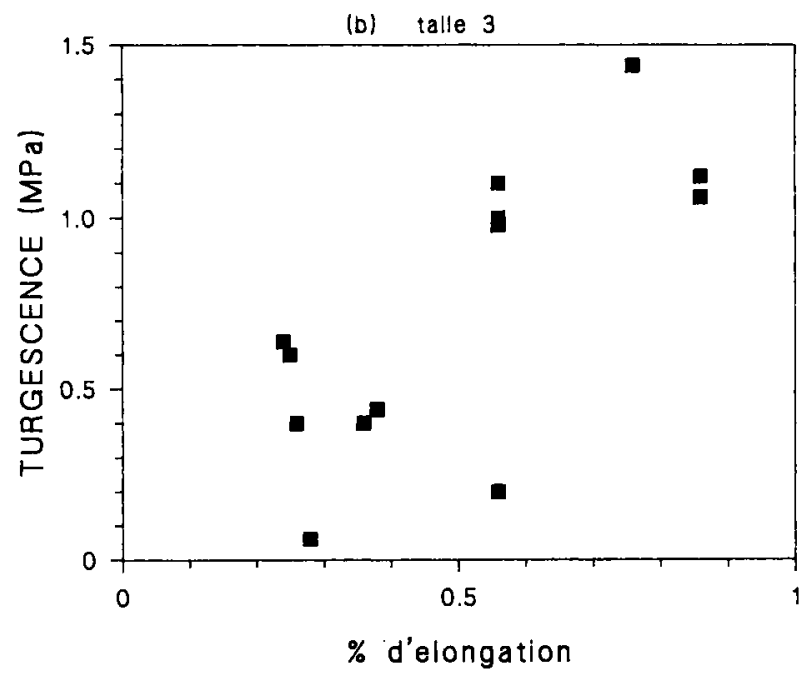

Fig 1. Évolution du potentiel de turgescence seuil $(Y$ ) avec la taille relative (en \% de la taille finale) de la dernière feuille en croissance du maître brin (a) et de la talle 3 (b). Chaque point représente une mesure indépendante. 
Tableau IV. Évolution des potentiels hydriques et osmotiques (MPa) au cours du tallage et de la montaison de plantes de blé à 2 densités de peuplement. La somme de température $\left({ }^{\circ} \mathrm{j}\right)$ est estimée, (base $0^{\circ} \mathrm{C}$ ) à partir de la levée. On a comparé les résultats obtenus pour les maîtres brins (MB) et les talles $3\left(T_{3}\right)$. L'intervalle de confiance à $5 \%, 10(150$ plantes $\left.\mathrm{m}^{-2}\right)$ et $40\left(300\right.$ plantes $\left.\mathrm{m}^{-2}\right)$ répétitions; * : différence significative à $5 \%$.

\begin{tabular}{|c|c|c|c|c|c|c|c|c|}
\hline \multirow[t]{3}{*}{$\left({ }^{\circ} \mathrm{C} \cdot j\right)$} & \multicolumn{4}{|c|}{$150 \mathrm{pl} / \mathrm{m}^{2}$} & \multicolumn{4}{|c|}{$300 \mathrm{pl} / \mathrm{m}^{2}$} \\
\hline & \multicolumn{2}{|c|}{ Phydr (MPa) } & \multicolumn{2}{|c|}{ Posm (MPa) } & \multicolumn{2}{|c|}{ P hydr (MPa) } & \multicolumn{2}{|c|}{ P osm (MPa) } \\
\hline & $M B$ & $T_{3}$ & $M B$ & $T_{3}$ & $M B$ & $T_{3}$ & $M B$ & $T_{3}$ \\
\hline 611 & $-1,3 \pm 0,15$ & & $-1,5^{a} \pm 0,22$ & $-1,0^{b} \pm 0,15$ & $-1,0 \pm 0,09$ & $-0,7 \pm 0,15$ & $-1,4^{a} \pm 0,09$ & $-1,1^{b} \pm 0,06$ \\
\hline 695 & $-0,8 \pm 0,15$ & $-0,6 \pm 0,07$ & $-1,6^{c} \pm 0,22$ & $-1,3^{d} \pm 0,15$ & $-0,7 \pm 0,09$ & $-0,3 \pm 0,03$ & $-1,4^{c} \pm 0,06$ & $-1,1^{d} \pm 0,06$ \\
\hline 755 & $-1,1 \pm 0,07$ & $-0,9 \pm 0,07$ & $-1,6^{c} \pm 0,22$ & $-1, \hat{j}^{\mathrm{d}} \pm 0,22$ & $-1,1 \pm 0,03$ & $-1,0 \pm 0,06$ & $-1,5^{c} \pm 0,06$ & $-1,2^{d} \pm 0,03$ \\
\hline 833 & $-1,0 \pm 0,15$ & $-0,9 \pm 0,15$ & $-1,69 \pm 0,15$ & $-1,2^{\mathrm{h}} \pm 0,07$ & $-1,0 \pm 0,06$ & $-0,8 \pm 0,06$ & $-1,49 \pm 0,09$ & $-1,2^{h} \pm 0,03$ \\
\hline 900 & $-0,3 \pm 0,07$ & $-0,4 \pm 0,07$ & $\pm 1,1^{i} \pm 0,15$ & $-1,1^{i} \pm 0,6$ & $-0,7 \pm 0,06$ & $-0,7 \pm 0,06$ & $-1,3^{i} \pm 0,09$ & $-1,1^{i} \pm 0,25$ \\
\hline 971 & $-1,0 \pm 0,07$ & $-1,0 \pm 0,07$ & $-1,5 \mathrm{j} \pm 0,37$ & $-1,2^{k} \pm 0,15$ & $-0,9 \pm 0,06$ & $-0,8 \pm 0,06$ & $-1,2^{k} \pm 0,06$ & $-1,1^{1} \pm 0,28$ \\
\hline 112 & $-1,0 \pm 0,15$ & $-0,8 \pm 0,07$ & $-1,3^{\prime} \pm 0,3$ & $-1,11 \pm 0,07$ & $-1,0 \pm 0,06$ & $-0,8 \pm 0,06$ & $-1,1 \mathrm{~m} \pm 0,06$ & $-1,0^{m} \pm 0,06$ \\
\hline
\end{tabular}

au cours du tallage et de la montaison de plantes de plein champ à 2 densités de culture. Le potentiel hydrique moyen en milieu de jour est de $-0,8 \mathrm{MPa}$. Les variations du potentiel hydrique sont dues à la très forte liaison entre ce paramètre et les conditions climatiques au moment de la mesure. L'ensemble des observations montre que la turgescence est toujours très positive : elle se maintient autour de $0,4-0,5$ $\mathrm{MPa}$. Des différences significatives entre $\mathrm{MB}$ et $T_{3}$ apparaissent, $T_{3}$ est en général dans un état hydrique plus favorable que MB. Aux 2 densités de culture, le potentiel osmotique de $T_{3}$ est toujours supérieur à celui de $\mathrm{MB}$, de $0,2 \mathrm{MPa}$ en moyenne (tableau IV).

Les molécules dosées dans la sève extraite des limbes en croissance, expliquent 50 à $55 \%$ de la pression osmotique mesurée. Une analyse de covariance des pressions osmotiques et des molécules dosées sur la totalité de l'essai montre que la variabilité des pressions osmotiques est pratiquement totalement expliquée par celle des concentrations des molécules choisies (Barcelo, 1987). Leur évolution au cours de la saison est rapportée figure 2.

Le potassium est l'élément majeur $(40 \%$ environ) expliquant la pression osmotique. Si l'on considère les anions qui doivent l'accompagner et dont seul le nitrate a été dosé, les sels de potassium et le glucose expliquent $90 \%$ de la pression osmotique. Au cours de la saison, on note une évolution opposée entre les teneurs en ions minéraux et le glucose. Du tallage à la montaison, la contribution des ions minéraux à la pression osmotique augmente. Par la suite, cette contribution diminue, contrebalancée partiellement par une augmentation de la teneur en glucose. La différence de pression osmotique entre $M B$ et $T_{3}$ se retrouve au début de la croissance sous forme de différence de concentrations en ions minéraux et à partir de la montaison sous forme de différence de teneur en sucres.

L'analyse en composantes principales de ces données montre que, du tallage à la montaison, les variations de pression osmotique au cours de la saison sont expliquées principalement par les variations des teneurs en ions minéraux. Inversement, à partir de la montaison, ces variations sont expliquées principalement par celle des sucres. Le peuplement en densité faible présente le même comportement (Barcelo, 1987).

\section{Évolution de la population de $\mathbf{T}_{3}$ en fin de montaison}

Au cours des 2 derniers prélèvements, nous avons observé dans les parcelles à forte densité 

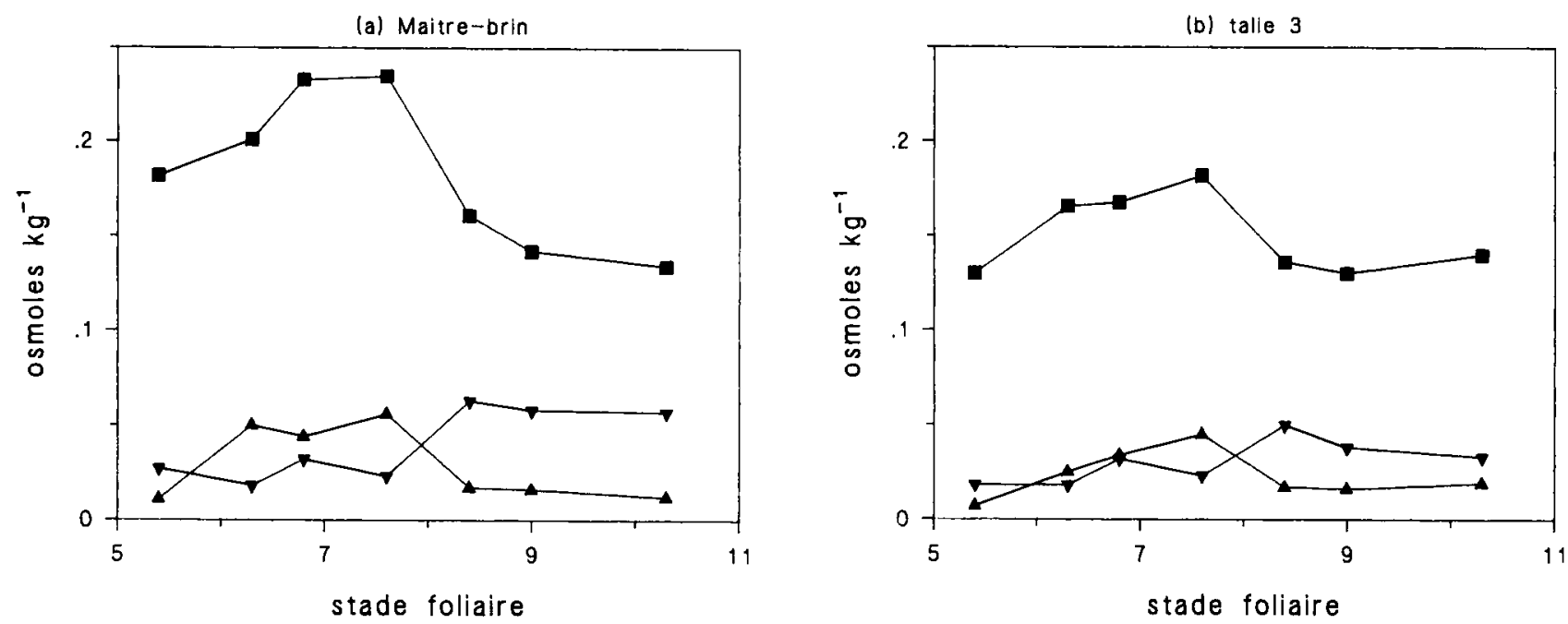

Fig 2. Évolution des principales composantes de l'osmolarité au cours du tallage et de la montaison dans les zones en croissance du maître brin (a) et de la talle 3 (b) : $\mathrm{K}^{+}: \square-\square, \mathrm{NO}_{3}^{--}: \mathbf{\Delta - \Lambda}$, glucose : $\mathbf{\nabla - \nabla}$.

de peuplement que des $T_{3}$ présentent des symptômes de régression (jaunissement et flétrissement). Ces symptômes accompagnent l'arrêt de croissance de certaines $T_{3}$ relativement aux $M B$ (fig 3) et du développement (mesuré par le stade foliaire) (tableau V). Le processus régressif apparaît de façon brutale entre 900 et $970{ }^{\circ} \mathrm{C} \cdot \mathrm{j}^{-1}$ pendant la montaison du $\mathrm{MB}\left(5^{\mathrm{e}}\right.$ et $6^{\mathrm{e}}$ prélèvements, fig 3). La fréquence choisie des échantillonnages n'a pas permis de mettre en évidence de façon anticipée, une population de $T_{3}$ destinée à régresser. Les potentiels hydrique et osmotique ne varient pas lors de la régression des

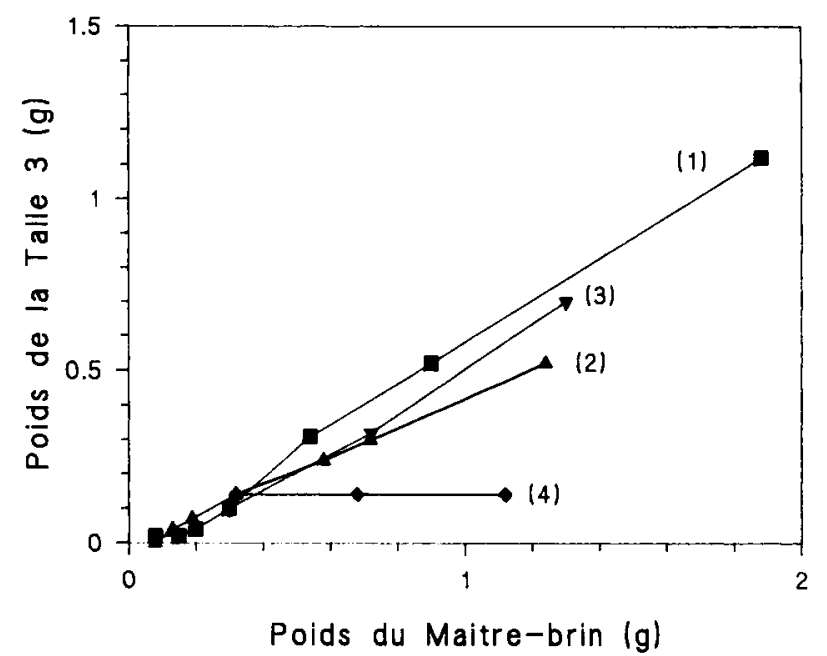

Fig 3. Relations entre les masses de matière sèche du maître brin et de la talie 3 , pour differentes densités de semis et différentes populations de plantes. Témoin semé à $150 \mathrm{pl} \cdot \mathrm{m}^{-2}$ : - $T_{3}$ sénescente $\left(300 \mathrm{pl} \cdot \mathrm{m}^{-2}\right): \nabla-\nabla$; plante à $T_{3}$ en croissance $\left(300 \mathrm{pl} \cdot \mathrm{m}^{-2}\right): \leftrightarrow$.
$T_{3}$ (tableau $V$ ). Par contre, on note un enrichissement très important en nitrates des talles en régression, ce qui explique le maintien de la pression osmotique.

\section{DISCUSSION}

L'entrée de l'eau est 1 des facteurs déterminants de la croissance des tissus. Elle se fait selon un gradient de potentiel entre le xylème et les tissus en croissance. Ce gradient a été mesuré expérimentalement (Cavalieri et Boyer, 1982; Wesgate et Boyer, 1985; Riazi et al, 1985). II est de environ $0,3 \mathrm{MPa}$ lorsque les tissus sont en équilibre hydrique et que la transpiration est nulle. Son origine est controversée : peut-être essentiellement une faible conductance hydraulique des tissus en croissance (Nonami et Boyer, 1987 ) ou la relaxation des parois cellulaires (Cosgrove, 1986).

En conditions inductrices de transpiration, le potentiel hydrique des tissus en croissance du MB du blé est très stable (tableau la), alors que celui des tissus adultes est beaucoup plus variable. II semble donc que les tissus engainés qui montrent toujours, dans nos conditions d'expérience, un potentiel hydrique plus élevé que les limbes dégainés, aient une alimentation en eau privilégiée, peut-être en raison d'une conductance hydraulique élevée des tissus séparant la racine des zones en croissance. Cette répartition prioritaire de l'eau vers les zones en croissance rend bien compte de l'indépendance 
Tableau V. Caractéristiques agronomiques, hydriques et composantes osmotiques des talles $3\left(\mathrm{~T}_{3}\right), 1112^{\circ} \mathrm{j}$ après la levée dans une culture à la densité de 300 plantes par $\mathrm{m}^{2}$. $^{\star}$ : différence significative à $5 \%$ (test de Wilcoxson, 12 répétitions pour les $T_{3}$ en montaison et 8 répétitions pour les $T_{3}$ en régression).

\begin{tabular}{lrllllll}
$\begin{array}{l}\text { Type } \\
\text { de T3 }\end{array}$ & $\begin{array}{c}\text { Stade } \\
\text { foliaire }\end{array}$ & Poids sec $(\mathrm{mg})$ & Phydr $(\mathrm{MPa})$ & Posm $(\mathrm{MPa})$ & \multicolumn{2}{c}{ Comp osm } & $\left(\mathrm{mOsm} \cdot \mathrm{kg}^{-1}\right)$ \\
& & & $\mathrm{K}^{+}$ & $\mathrm{NO}_{3}^{-}$ & Fru Glu \\
\hline
\end{tabular}

$\mathrm{T}_{3}$ montante

\begin{tabular}{|c|c|c|c|c|}
\hline $5,5 \pm 1,3$ & $739 \pm 72,3$ & $\begin{array}{c}-1,04 \pm 0,5 \\
\text { NS }\end{array}$ & $\begin{array}{c}-0,93 \pm 0,12 \\
\text { NS }\end{array}$ & $\begin{array}{c}132 \pm 19 \\
\text { NS }\end{array}$ \\
\hline
\end{tabular}

$\mathrm{T}_{3}$ en régression

$4 \pm 0,4 \quad 186 \pm 67 \quad-0,85 \pm 0,1 \quad-0,68 \pm 0,1 \quad 143 \pm 16 \quad 30 \pm 11 \quad 2,2 \pm 1,5 \quad 11,2 \pm 5,1$

relative de l'allongement du limbe de blé par rapport à la transpiration. L'allongement varie peu en particulier, en fonction des alternances journuit (Christ, 1978), alors que la croissance diurne des limbes de dicotylédones est très ralentie (Bunce, 1977).

Néanmoins, nos résultats sont différents de ceux de Michelena et Boyer (1982) qui, sur maïs, observent un potentiel hydrique plus faible dans les zones en croissance. De même, Barlow (1986) constate que les zones en allongement ont des pressions de turgescence plus faibles que les zones adultes.

D'autre part, nous observons, comme Barlow (1986), une absence de gradient de potentiel osmotique le long de la feuille. Des différences de potentiel hydrique entre zones engainées et dégainées sont donc dues à des différences de potentiel de turgescence entre ces même zones.

La zone dégainée de $T_{3}$ est plus sensible à la transpiration diurne, son potentiel hydrique diminue relativement plus que celui du MB. Cependant la pression de turgescence au milieu de la journée reste importante (1 $\mathrm{MPa}$, tableau I) et semble suffisante pour maintenir la croissance. On ne peut pas déterminer si cette différence de comportement hydrique de $\mathrm{MB}$ et $\mathrm{T}_{3}$ peut intervenir sur leurs vitesses de croissance relative au champ.

Le potentiel seuil de turgescence est une caractéristique déterminante de la vitesse de croissance (Wesgate et Boyer, 1985). II pourrait déterminer la répartition de la croissance entre les différentes parties de la plante. La diminution de la surface d'échange des racines avec la solution nutritive (expérience 1) ne conduit à un arrêt de croissance que lorsque la surface d'absorp- tion est réduite à 0 , alors que la turgescence des feuilles en croissance n'est pas encore significativement affectée. On peut conclure que si une certaine turgescence positive est une condition nécessaire à la croissance, d'autres phénomènes physiologiques peuvent la bloquer (Mathews et al, 1984). Cependant, la compartimentation cellulaire de la pression de turgescence pourrait ne pas être détectée par nos méthodes globales de mesure du potentiel osmotique. Enfin, il est possible que le stress hydrique appliqué pendant cette expérience de courte durée (environ $1 \mathrm{~h}$ ), ne permet pas de réaliser dans la plante un équilibre hydrique stable (Boyer et al, 1985) et altère les mesures de pression de turgescence.

La croissance semble répondre instantanément aux modifications des conditions hydriques des racines. La résistance au flux d'eau entre les racines et les feuilles serait donc faible. Les conditions de culture hydroponique produisent des plantes à faible conductance hydraulique (Morizet et al, 1988). Par contre, le gradient de potentiel hydrique entre tissus engainés et dégainés suggère une faible conductance hydraulique entre ces tissus.

La technique de manipulation de la pression de turgescence des tissus excisés en croissance de Cleland (1971) permet de mesurer une valeur moyenne du potentiel de turgescence seuil de $1 \pm 0,2$ pour le $\mathrm{MB}$ avec une bonne précision. Cette valeur est comparable à celles déjà publiées (Glass, 1983). Elle est indépendante de l'âge du limbe en croissance. Par contre les $T_{3}$ présentent un potentiel seuil variable avec leur âge ou leur taille. Les jeunes talles $\mathrm{T}_{3}$ ont un potentiel seuil faible qui augmente ensuite pour at- 
teindre celui du MB lorsqu'ils atteignent $60 \%$ de leur longueur finale. Cette caractéristique permet d'envisager un comportement différencié de MB et $T_{3}$. La croissance des jeunes $T_{3}$ sera relativement moins sensible au déficit hydrique que celle de MB. Ces différences devraient être confirmées sur des plantes développées au champ puisque $Y$ dépend des conditions de culture (Tyree et Jarvis, 1982; Mathews et al, 1984; Barlow, 1986).

Dans les conditions variables du champ, la turgescence seuil serait plus souvent dépassée et permettrait la croissance de $T_{3}$ et donc, la durée de croissance autorisée sera plus longue. Cette caractéristique pourrait contribuer à l'homogénisation pour une plante de blé de la taille de ses talles, dont la croissance ne démarre pas à la même date (Masle-Meynard, 1981a).

Les mesures des paramètres hydriques des $M B$ et $T_{3}$ au champ pendant la montaison montrent que les talles $T_{3}$ en situation de compétition (densité de semis : 300 plantes $\cdot \mathrm{m}^{-2}$ ) présentent des potentiels hydriques moyens plus élevés, donc des potentialités de croissance supérieures ou égales à celles du MB. Par contre, la pression osmotique de $\mathrm{MB}$ est supérieure en moyenne à celle de $T_{3}$. II est possible que ce soit un effet de l'âge de la talle ou de l'ordre de la feuille en croissance (Morgan, 1984). Cette différence augmente la capacité de résistance à un déficit hydrique, de $M B$, en permettant le maintien de la turgescence à des potentiels hydriques plus bas.

L'osmolarité est assurée en majorité par les ions minéraux, mais on note une importance croissante des sucres au cours de la montaison (fig 2).

Le faible rôle joué par les sucres dans l'osmolarité des tissus en croissance peut s'expliquer par le fait que ces tissus sont activement consommateurs. On peut penser qu'il existe une compétition pour les sucres entre l'osmorégulation et la nutrition carbonée. Les travaux de Kemp et Blacklow (1980) montrent que des concentrations en sucres d'environ $10 \mathrm{mmol} / \mathrm{l}$ sont saturantes pour la croissance des feuilles de blé. Les concentrations que nous avons mesurées dans les zones en croissance des plantes de plein champ sont très supérieures (tableau IV). Nous concluons que la nutrition carbonée ne limitait pas la croissance des limbes pendant la montaison.

La régression des talles $T_{3}$ est un phénomène qui se manifeste brusquement en fin de montaison et nos mesures n'ont pas permis de le pré- voir. La pression osmotique des talles en cours de régression n'est pas modifiée et les concentrations en glucides restent saturantes pour la croissance. Le fonctionnement des tissus en croissance est donc bloqué par d'autres facteurs, comme l'activité métabolique (systèmes enzymatiques inhibés, Claire et Arnaudet, 1984) ou les caractéristiques biomécaniques pariétales (Cosgrove, 1986). De telles modifications pourraient être liées à la position des limbes adultes de $T_{3}$ placés dans un champ lumineux limitant et dont les caractéristiques spectrales actives sur l'équilibre phytochrome sont modifiées (Bleiss et al, 1987). II est cependant clair que l'arrêt de croissance des talles $T_{3}$ à forte densité de culture n'est pas dû à une limitation des apports des principaux éléments de la croissance : les molécules responsables de la pression osmotique, l'eau ou les glucides.

\section{REMERCIEMENTS}

Ce travail a été financé par le contrat 3 «Écophysiologie du blé», ONIC, INRA, ITCF.

\section{RÉFÉRENCES}

Barcelo MO (1987) Facteurs physiologiques déterminant la croissance des différentes talles de blé. Thèse Docteur-Ingénieur. INA PG

Barlow EWR (1986) Water relations of expanding leaves. Aust J Plant Physiol 13, 45-58

Bleiss W, Lorbeer $H$, Göring $H$ (1987) Rapid growth response of dark-grown wheat seedlings to red light-irradiation. I. Kinetic studies on the short-term growth responses of intact coleoptiles at different seedling age. Plant Cell Physiol 28, 253-262

Boyer JS, Cavalieri AJ, Schulze E (1985) Control of the rate of cell enlargment : excision wall relaxation on growth induced water potential. Planta 163, 527-543

Bunce JA (1977) Interrelation of diurnal expansion rates and carbohydrate accumulation and movement in soya beans. Ann Bot 42, 1463-1466

Cataldo DA, Haroon M, Schrader LE, Youngs VL (1975) Rapid colorimetric determination of nitrate in plant tissue by nitritation of salycilic acid. Commun Soil Sci Plant Anal 6, 71-80

Cavalieri AJ, Boyer JS (1982) Water potential induced by growth in soybean hypocotyls. Plant Physiol 69, 492-496

Christ RA (1978) The elongation rate of wheat leaves: elongation rates during day and night. $J$ Exp Bot $29,603-610$ 
Claire A, Arnaudet L (1984) Le métabolisme glucidique en relation avec la croissance et le mouvement révolutif des tiges volubiles d'lpomea purpurea. Physiol Vég 22, 405-413

Cleland RE (1971) Cell wall extension. Annu Rev Plant Physiol 22, 197-222

Coic $Y$, Lesaint $C$ (1973) La nutrition minérale en horticulture avancée. Rev Hortic 2316, 29-34

Cosgrove D (1986) Biophysical control of plant cell growth. Annu Rev Plant Physiol 36, 377-405

Cosgrove D, Cleland RE (1983) Solutes in the free space of growing stem tissues. Plant Physiol 72, 326-331

Fry SC (1986) Cross-linking of matrix polymers in the growing cell walls of Angiosperms. Annu Rev Plant Physiol 37, 165-233

Glass ADM (1983) Regulation of ion transport. Annu Rev Plant Physiol 34, 311-347

Handa S, Bressan RA, Handa AK, Carpita NC, Hasegawa PM (1983) Solutes contributing to osmotic adjustment in cultured plant cells adapted to water stress. Plant Physiol 73, 834-843

Katz A, Avron M (1985) Determination of intracellular osmotic volume and sodium concentration in Dunaliella. Plant Physiol 78, 817-820

Kemp DR (1981a) The growth rate of wheat leaves in relation to the extension zone sugar concentration manipulated by shading. $J$ Exp Bot $32,141-150$

Kemp DR (1981b) Comparison of growth rates and sugar, protein concentrations on the extension zone of main shoot and tiller leaves of wheat. $J$ Exp Bot 32, 151-158

Kemp DR, Blacklow WM (1980) Diurnal extension rates of wheat leaves in relation to temperature and carbohydrate concentration of the extension zone. $J$ Exp Bot 31, 821-828

Kemp DR, Whingwiri EE (1980) Effect of tiller removal and shading on spikelet development and yield components of the main shoot of wheat and on the sugar concentration of ear and flag leaf. Aust $J$ Plant Physiol 7, 501-510

Lockhart JA (1965) An analysis of irreversible plant cell elongation. J Theor Biol 8, 264-275

Masle-Meynard J (1981a) Relation entre croissance et développement pendant la montaison d'un peuplement de blé d'hiver. Influence des conditions de nutrition. Agronomie 1, 365-374

Masle-Meynard J (1981b) Élaboration du nombre d'épis d'un peuplement de blé d'hiver en situation de compétition pour l'azote. I. Mise en évidence d'un stade critique pour la montée d'une talle. Agronomie 1, 623-632
Matthews MA, Darvill AG, Fry SC, Albersheim P (1984) Structure and fonction of primary cell walls of plants. Annu Rev Biochem 53, 625-663

Michelena VA, Boyer JS (1982) Complete turgor maintenance at low water potentials in the elongating region of maize leaves. Plant Physiol 69, 1145-1149

Morgan JM (1984) Osmoregulation and water stress in higher plants. Annu Rev Plant Physiol 35, 299-319

Morizet J, Cruiziat P, Boussignac F (1988) Étude comparative du transfert hydrique dans les systèmes racinaires du tournesol et du maïs. Implications éventuelles concernant les consommations d'eau, $p$ 225-245. In : Études sur les transferts d'eau dans le système sol-plante-atmosphère ( $\mathrm{R}$ Calvet, ed) INRA, Paris, 362 pp

Nonami H, Boyer JS (1987) Origin of growth induced water potential: solute concentration is low in apoplast of enlarging tissues. Plant Physiol 88, 596-601

Riazi A, Matsuda K, Arsland A (1985) Water stress induced changes in concentrations of proline and other solutes in growing regions of young barley leaves. J Exp Bot 36, 1716-1725

Scholander PF, Hammel HT, Bradstreet ED, Hemmingsen EA (1965) Sap pressure in vascular plants. Science 148, 339-346

Silverstown JM (1982) Introduction to plant population ecology. Longman, Londres, $200 \mathrm{pp}$

Sweeley CC (1965) Analyse des hydrates de carbone par chromatographie en phase gazeuse. Bull Soc Chim Biol 47, 1477-1493

Taiz L (1984) Plant cell expansion : regulation of cell wall mechanical properties. Annu Rev Plant Physiol $35,585-657$

Tyree MT, Jarvis PG (1982) Water in tissues and cells. In: Physiological Plant Ecology. II. Water relations and carbon assimilation (OL Lange, PS Nobel, CS Osmon, $\mathrm{H}$ Ziegler, eds) Encyclopedia of Plant physiology. New series vol 12B Springler Verlag, Berlin 35-77

Tomos AD (1985) The physical limitation of leaf cell expansion. In: Control of leaf growth (NR Baker, WD Davies, C Ong, eds) Soc Biol Seminar Series 27. Cambridge, 1-33

Vesper MJ (1985) Use of a $\mathrm{pH}$ response-curve for growth to predict apparent wall $\mathrm{pH}$ in elongating segments of maize coleoptils and sunflower hypocotyls. Planta 166, 96-104

Wesgate ME, Boyer JS (1985) Osmotic adjustment and the inhibition of leaf, root, stem and silk growth at low water potentials in maize. Planta 164, 540. 549

Westoby M (1984) The self-thinning rule. Adv Ecol Res 14, 167-224 